\title{
金刚石基燃料电池催化剂的研究进展
}

\author{
董亮 ${ }^{1,2}$, 王艳辉 ${ }^{2}$, 㶓建兵 ${ }^{2}$
}

(1. 东北大学秦皇岛分校 河北省电介质与电解质功能材料重点实验室, 秦皇岛 $066004 ; 2$. 燕山大学 亚稳材料制备 技术与科学国家重点实验室, 秦皇岛 066004)

摘 要: 金刚石是由共价键方式连接的 $\mathrm{sp}^{3}$ 杂化碳原子组成, 具有极强的稳定性。含喼金刚石(BDD)薄膜、BDD 颗 粒、非掺杂纳米金刚石(ND)等新型金刚石又兼具一定的导电性，因此成为高稳定性燃料电池催化剂的理想载体材 料。研究者进一步发现通过对上述新型金刚石进行适当功能化处理, 可以进一步提高催化剂的催化活性和稳定性。 对金刚石进行掺杂处理，既包括向金刚石晶格中掺杂，也包括向金刚石衍生的石墨结构中进行掺杂，能够得到新 型高稳定性燃料电池非铂催化剂，且金刚石 $\mathrm{sp}^{3}$ 结构在提高非铂催化剂稳定性方面作用独特。本文总结介绍了相关 研究成果, 希望能为后续研究提供参考借鉴。

关 键 词: 金刚石; 燃料电池; 稳定性; 综述

中图分类号: TQ152 文献标识码: A

\section{Recent Progress in Diamond-based Electrocatalysts for Fuel Cells}

\author{
DONG Liang $^{1,2}$, WANG Yan-Hui ${ }^{2}$, ZANG Jian-Bing ${ }^{2}$
}

(1. Northeastern University at Qinghuangdao, Key Laboratory of Dielectric and Electrolyte Functional Material Hebei Province, Qinghuangdao 066004, China; 2. Yanshan University, State Key Laboratory of Metastable Materials Science and Technology, Qinghuangdao 066004, China)

\begin{abstract}
Attributed to highly stable structure of $\mathrm{sp}^{3}$ hybridized carbon atoms, diamond has excellent physical and chemical stabilities. As new conductive diamond materials, boron-doped diamond (BDD) films and particles, as well as undoped nanodiamond (ND) has become the ideal support of the high stability electrocatalysts for fuel cells. Futher investigation showed that the activity and stability of electrocatalysts could be futher improved if the new diamond materials were properly processed. The doping treatment, including doping into diamond and the graphite structure from conversion of diamond, was used to produce diamond-based non-Pt electrocatalysts for fuel cells. It was considered that the $\mathrm{sp}^{3}$ structure of diamond played a unique role in enhancing the stability of diamond-based non-Pt electrocatalysts. In this paper, related studies of diamond-based electrocatalysts were summarized for the references of future study.
\end{abstract}

Key words: diamond; fuel cell; durability; review

燃料电池是广受关注的新能源技术，近些年 来已经从航空和发电站领域向汽车、军事、电子设
备等领域渗入，且商业化程度越来越高。本文讨论 的燃料电池主要是氢气燃料电池和直接醇类燃料

收稿日期：2016-08-29; 收到修改稿日期：2016-11-10

基金项目: 国家自然科学基金(51602043); 中央高校基本科研业务费(N152303001); 河北省高等学校科学技术研究项目 (QN2015315); 东北大学秦皇岛分校校内科研基金(XNB201624)

National Natural Science Foundation of China (51602043); Fundamental Research Funds for the Central Universities (N152303001); Foundation for Science and Technology Research in Higher Education of Hebei (QN2015315); Research Funds of Northeastern University at Qinhuangdao (XNB201624)

作者简介: 董 亮(1985-), 男, 讲师. E-mail: dongliang@neuq.edu.cn 
电池 ${ }^{[1-3]}$, 其中氢气燃料电池的能量密度高, 对环境 友好, 并已经应用到量产的燃料电池汽车上。直接 醇类燃料电池可以在低温下运行, 在电子设备、军 事等领域有十分广阔应用前景 ${ }^{[4-5]}$ 。在燃料电池商业 化道路上, 始终面临催化剂稳定性差、成本高昂等 问题。因此, 提高催化剂稳定性, 降低催化剂制备成 本的相关研究一直是热点内容。

商用燃料电池催化剂主要是碳载(如炭黑 $\mathrm{XC}-72)$ 铂基催化剂 $(\mathrm{Pt} / \mathrm{C})$ 。 $\mathrm{Pt} / \mathrm{C}$ 的稳定性较差, 是燃 料电池运行时长不理想的重要原因 ${ }^{[6]}$ 。导致 $\mathrm{Pt} / \mathrm{C}$ 稳 定性差的主要因素之一就是炭黑等无定型碳载材料 在燃料电池运行过程中容易被氧化。如图 1 所示, 碳 载被氧化之后, 其结构发生塌陷, 导致负载的纳米 铂颗粒(Pt-NPs)流失或团聚, 使得催化剂失活 ${ }^{[7-8]}$ 。

因此结晶度高、抗氧化能力强的新型碳材料开始被 应用于该领域。这些碳材料包括零维的碳量子点 ${ }^{[9]}$ 、 一维的碳纳米管 ${ }^{[10]}$ 、二维的石墨烯 ${ }^{[11-15]}$ 和三维的碳 泡沫 ${ }^{[16-17]}$ 等等。

金刚石也是碳材料的一种, 其中的碳原子发生 $\mathrm{sp}^{3}$ 杂化, 互相以共价单键方式结合, 形成空间正四 面体结构。这种稳定的晶体结构, 使其具有极强的抗 氧化能力。金刚石在室温环境下不会被任何酸碱腐 蚀, 在纯氧中开始氧化的温度也高达 $720 \sim 800^{\circ} \mathrm{C}^{[18]}$ 。 这些特点使得金刚石成为替代碳载的热门材料, 用 于制备了铂、铂合金及含铂双金属催化剂。研发合 金或者双金属催化剂的目的主要是提高催化剂的催 化性能，降低贵金属铂的用量，节约制备成本。

$\mathrm{Pt} / \mathrm{C}$ 稳定性差的因素还包括: 1) 对于直接醇类 燃料电池而言, 醇类的氧化反应有时会产生中间产 物 CO, 它会在铂颗粒表面以 $\sigma-\pi$ 键形式形成强力 吸附, 占据催化活性位, 导致催化剂 CO 中毒 ${ }^{[19-20]}$; 2) Pt 与无定型的碳载之间结合力较差, Pt-NPs 受表 面能最小化趋势影响, 会发生迁移、团聚甚至烧结,
使催化剂失活 ${ }^{[21-22]}$ 。对金刚石进行表面处理，使其 表面功能化, 能够提高催化剂的抗 $\mathrm{CO}$ 中毒的能力, 以及载体与 Pt-NPs 之间结合力, 同时保持金刚石 高稳定性的优势, 进而能够进一步提高催化剂的 稳定性。

另外, $\mathrm{Pt}$ 是稀有金属, 价格十分高昂。根据相关 统计结果，使用 $\mathrm{Pt}$ 的花费占燃料电池总成本的 $30 \% \sim 50 \%{ }^{[23]}$ 。因此, 研发非铂催化剂是推动燃料电 池最终商业化的核心技术之一。目前, 主要非铂催 化剂包括过渡族金属大环化物、杂原子掺杂的碳材 料、过渡族金属碳化物或者它们共同组成的复合材

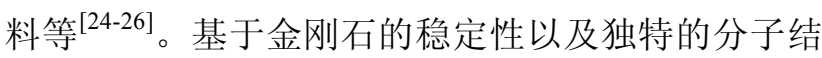
构, 它也常常被用于研发制备非铂催化剂, 并在稳 定性方面有独特优势。本文归纳分析了金刚石在燃 料电池领域的应用研究进展, 讨论了它的突出优势 以及相关研究发展方向, 为后续相关研究工作的开 展提供借鉴。

\section{1 金刚石基铂及合金、双金属催化剂}

传统大尺寸金刚石是绝缘体，以其为载体会影 响催化剂的活性。但含硼金刚石(BDD)薄膜、BDD 颗粒、非掺杂纳米金刚石(ND)等新型金刚石类材料 兼具高稳定性和一定导电性, 是燃料电池催化剂较 为理想的载体材料。

\section{1 以含嗍金刚石薄膜为载体}

以嗍原子替代碳原子, 对金刚石进行嗍掺杂处 理，会使金刚石的晶格中形成空穴载流子，进而使 金刚石获得一定导电性。BDD 也是最为常见、最早 被应用的金刚石类载体材料，具有背景电流低、电 势窗口宽等特点。Swain 等 ${ }^{[27]}$ 在硝酸和氟化钠混合 溶液中对 BDD 进行 $2 \mathrm{~h}$ 的电化学氧化处理, 通过表 征测试发现 BDD 表面既没有出现结构塌陷, 也没有

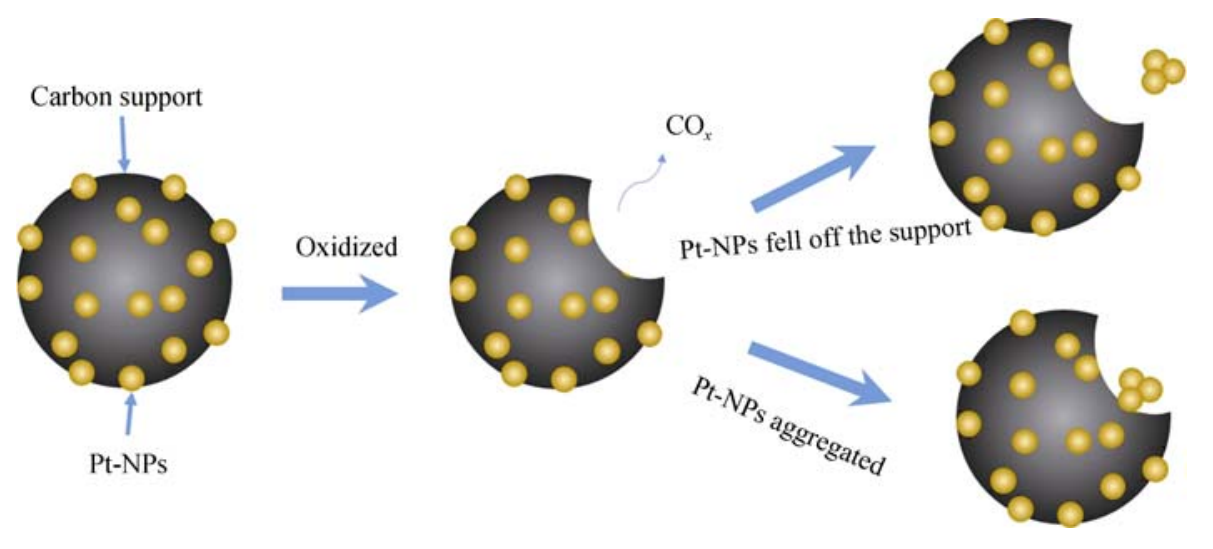

图 1 碳载被氧化导致催化剂失活的示意图

Fig. 1 Schematic illustration of losing activity the catalysts by oxidized carbon support 
含氧物质, 表现出极强的稳定性。基于这一巨大优 势, 研究者们纷纷以 BDD 薄膜为载体材料, 利用电 沉积 ${ }^{[28-30]}$ 、微乳液 ${ }^{[31]}$ 、溶胶-凝胶 ${ }^{[32]}$ 等零维纳米颗 粒制备方法，成功获得 Pt、PtRu 等合金以及 Pt-Au、 $\mathrm{Pt}-\mathrm{Cu} 、 \mathrm{Pt}-\mathrm{Sn}$ 双金属催化剂负载的燃料电池电极催 化剂。Wang 等 ${ }^{[33]}$ 以 $\mathrm{H}_{2}$ 为还原气体, 利用微波辅助 的化学气相沉积技术(CVD)将 10 500 nm 尺寸的 Pt 颗粒沉积到 BDD 表面, 该催化剂稳定性十分出色, 并具有优异的对甲醇氧化反应(MOR)的催化活性。

但该报道中 Pt-NPs 的尺寸范围过大 $(10 \sim 500 \mathrm{~nm})$, 且分布不均匀。Gao 等 ${ }^{[28]}$ 通过两步法在 BDD 表面 均匀负载了 Pt-NPs, 实现对 Pt-NPs 尺寸的调控, 并 通过热处理, 使 Pt-NPs 与 BDD 之间形成较为牢固 的偶极矩间作用力, 进一步提高催化剂的稳定性。 Lyu 等 ${ }^{[34]}$ 基于化学镀技术, 开发了一种更为绿色简 单的铂基催化剂制备方法, 并通过调节反应温度和 溶液 $\mathrm{pH}$ 的方式, 实现对 Pt-NPs 分散均匀性的调控。

$\mathrm{Pt}$ 基合金或者双金属催化剂既有利于降低催化 剂成本, 也能够提高催化剂性能, 而以 BDD 为载体, 能够显著提高催化剂的稳定性。如 Tryk 等 ${ }^{[35]}$ 和 $\mathrm{Lu}$ 等 ${ }^{[29]}$ 均制备了稳定性优异的 PtRu/BDD, 并且 $\mathrm{Lu}$ 等 发现当 $\mathrm{Pt}$ 与 $\mathrm{Ru}$ 的原子比为 0.29 时催化活性最佳。 Comninellis 等 ${ }^{[31]}$ 采用微乳液法制备 BDD 负载的 Pt-Sn 双金属催化剂, 用于催化乙醇氧化, 他们发现 $\mathrm{Pt}_{80} \mathrm{Sn}_{20}$ 的催化活性最好, 且抗 $\mathrm{CO}$ 中毒能力更强。 可见采用 BDD 做载体, 催化剂的稳定性十分优异。 Foord 等 ${ }^{[30]}$ 认为 BDD 出色的稳定性, 适合做燃料电 池催化剂的载体, 并通过分步电沉积法制备了 $\mathrm{Pt}-\mathrm{Cu}$ 双金属催化剂。因为 $\mathrm{Pt}$ 与 $\mathrm{Cu}$ 之间协同作用, $\mathrm{Pt}-\mathrm{Cu} / \mathrm{BDD}$ 具有比 $\mathrm{Pt} / \mathrm{BDD}$ 更高的 MOR 催化活性以 及更少的表面污染。Roustom 等 ${ }^{[36]}$ 利用磁控溅射沉 积 $\mathrm{Au}$ 纳米颗粒到 BDD 表面, 再通过电沉积负载 Pt, 形成双金属催化剂 Pt-Au/BDD, 可用于催化燃料电 池阴极的氧还原反应(ORR)。Pt 的含量影响催化 ORR 的起始电位和反应电子数, 其中 $80 \mathrm{Pt}-20 \mathrm{Au} /$ BDD 活性最好, 起始电位 $0.78 \mathrm{~V}(\mathrm{vs} . \mathrm{SHE})$, 反应电 子数趋近于 4 。

将纳米氧化物修饰到 BDD 表面, 获得的新载 体材料, 既保持了 BDD出色稳定, 还能够与 Pt之间 同样存在协同催化作用。如 Machado 等 ${ }^{[32]}$ 将 $\mathrm{SnO}_{2}\left(\mathrm{Ta}_{2} \mathrm{O}_{5}\right)$ 与 Pt-NPs 共沉积到 BDD 表面, 制备得 到 Pt- $\mathrm{SnO}_{2} / \mathrm{BDD}\left(\mathrm{Pt}-\mathrm{Ta}_{2} \mathrm{O}_{5} / \mathrm{BDD}\right)$, 并发现 BDD 对催 化乙醇和甲醇氧化具有独特优势, 其独自催化的起 始电位在 1.49 和 $1.54 \mathrm{~V}$ (vs. SHE), 对提高催化剂催 化活性可能有一定帮助。修饰氧化物以后, 催化剂
抗 $\mathrm{CO}$ 中毒能力获得提升。同时 Machado 等还发现 $\mathrm{Pt}-\mathrm{Ta}_{2} \mathrm{O}_{5} / \mathrm{BDD}$ 更适合催化甲醇, $\mathrm{Pt}-\mathrm{SnO}_{2} / \mathrm{BDD}$ 更适 合催化乙醇。Spătaru 等 ${ }^{[37]}$ 采用两步法分别将 $\mathrm{RuO}_{X} \cdot n \mathrm{H}_{2} \mathrm{O}$ 和 Pt-NPs 负载到 BDD 表面, 制备得到 $\mathrm{Pt}-\mathrm{RuO}_{x} \cdot n \mathrm{H}_{2} \mathrm{O} / \mathrm{BDD}$, 发现氧终止处理 BDD, 可以使 $\mathrm{RuO}_{x} \cdot n \mathrm{H}_{2} \mathrm{O}$ 的载量从 $3 \mu \mathrm{g} / \mathrm{cm}^{2}$ 提高到 $140 \mu \mathrm{g} / \mathrm{cm}^{2}$ 。 电催化性能分析表明 BDD 提高了催化剂的稳定性, $\mathrm{RuO}_{x} \cdot n \mathrm{H}_{2} \mathrm{O}$ 提高了催化剂对 MOR 的催化活性。

\section{2 以含嗍金刚石颗粒为载体}

薄膜状 BDD 做载体优势十分明显, 但其比表 面积过低。针对这个问题, 不同研究者给出了一些 解决方案。Avaca 等 ${ }^{[38]}$ 以 $\mathrm{RuO}_{\mathrm{x}}$ 修饰的颗粒状 $\mathrm{BDD}$ 为载体制备得到 $\mathrm{Pt}-\mathrm{RuO}_{\mathrm{x}} / \mathrm{BDD}$, 并发现 $\mathrm{Pt}-\mathrm{RuO}_{\mathrm{x}} /$ $\mathrm{BDD}$ 催化 MOR 反应的起始电位比 $\mathrm{Pt} / \mathrm{BDD}$ 更低, 低 约 $20 \mathrm{mV}$ 。Spătaru 等 ${ }^{[39]}$ 从元素六公司购得粒径小于 $150 \mu \mathrm{m}$ 的 BDD 制备 Pt/BDD。Pt/BDD 的稳定性优于 $\mathrm{Pt} / \mathrm{C}$, 且以 $\mathrm{BDD}$ 为载体, 可能会降低 $\mathrm{CO}$ 对 Pt-NPs 的毒化作用。不过, 球磨法制备出的 BDD 颗粒粒径 均在微米级别, 比表面积的增加有限。Cabrera 等 ${ }^{[0]}$ 利用电场增强扩散技术在纳米非掺杂金刚石(ND)表 面掺入 $\mathrm{B}$ 原子形成 BDD 壳层, 制备得到纳米级的颗 粒状 ND@BDD。通过硝酸溶液清洗, 可以增加 ND 和 ND@BDD 表面含氢官能团。化学沉积 Pt 和 PtRu 后，他们发现金属纳米颗粒易于在吸附 $\mathrm{OH}$ 的 $\mathrm{BDD}$ 表面形核。Kim 等 ${ }^{[41]}$ 研究更加深入, 他们通过结合 静电自组装和 CVD 技术, 制备出纳米级 ND@BDD 颗粒。由于以纳米尺寸的 BDD 颗粒为载体, Pt/BDD 的活性比表面积(ESA)略大于 $\mathrm{Pt} / \mathrm{C}$ 和 $\mathrm{Pt} / \mathrm{CNT}$ 。通过 $100 \mathrm{~h}$ 稳定性测试以后, Pt 在 BDD 表面仅仅损失 $30 \%$, 而在 XC-72 和 CNT 表面流失掉 70\%和 $60 \%$, 即使在单电池测试中, $\mathrm{Pt} / \mathrm{BDD}$ 的稳定性也明显优于 $\mathrm{Pt} / \mathrm{C}$ 和 $\mathrm{Pt} / \mathrm{CNT}$ 。Celorrio 等 ${ }^{[42]}$ 制备了氧终止纳米 $\mathrm{BDD}$ 颗粒(ODP)和氢终止纳米 BDD 颗粒(HDP), 然 后进行载铂, 制备得到 $\mathrm{Pt} / \mathrm{HDP}$ 和 $\mathrm{Pt} / \mathrm{ODP}$ 。如图 2 所示, $\mathrm{Pt} / \mathrm{HDP}$ 和 $\mathrm{Pt} / \mathrm{ODP}$ 对吸附的 $\mathrm{CO}$ 的氧化电位, 相较于 $\mathrm{Pt} / \mathrm{C}$ 负向移动 $100 \mathrm{mV}$, 即 $\mathrm{CO}$ 在它们表面更 易于被氧化。他们认为通过对 BDD 表面进行不同 处理、调整处理程度以及改变 $\mathrm{B}$ 掺杂水平, 都将影 响 BDD 表面电子结构, 进而满足其在储能领域不 同的应用需要。除了考虑在 ND 表层沉积 $\mathrm{BDD}$, 还 有研究者 ${ }^{[43}$ 将 BDD 沉积到载铂之后的 BDD 表面, 固定 Pt-NPs。通过在高氯酸和高温磷酸 $\left(170^{\circ} \mathrm{C}\right)$ 中的 稳定性测试，Wang 等 ${ }^{[43]}$ 发现测试前后催化剂对 ORR 和析氢反应(HER)的活性几乎没有损失。但该 研究要将 Pt-NPs 半埋入 BDD 中, 显著减少了催化 


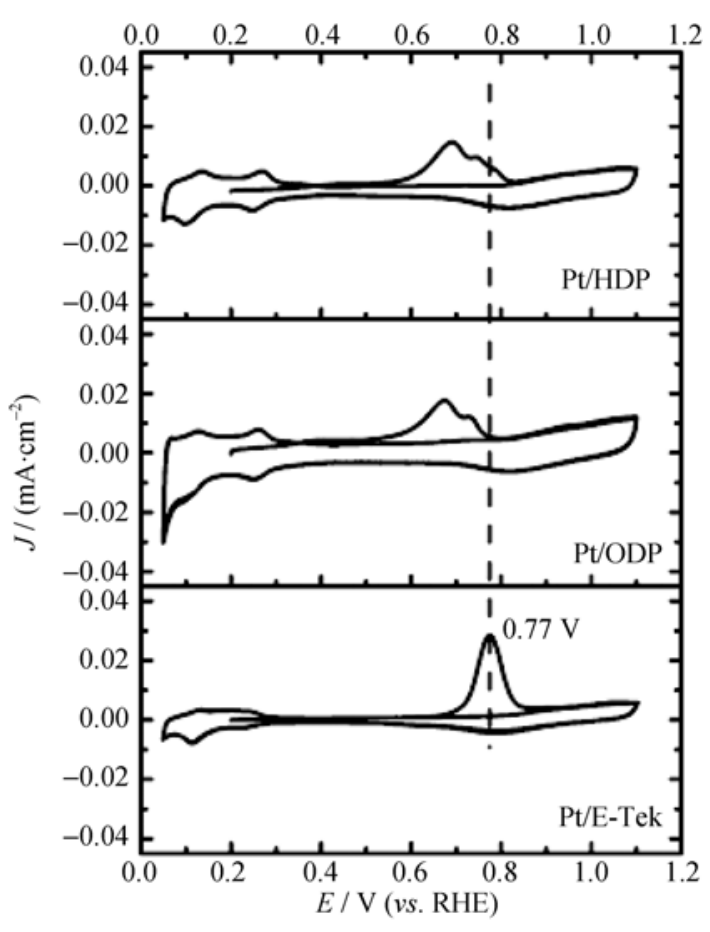

图 $2 \mathrm{Pt} / \mathrm{C}(\mathrm{Pt} / \mathrm{E}-\mathrm{Tek}) 、 \mathrm{Pt} / \mathrm{HDP}$ 和 $\mathrm{Pt} / \mathrm{ODP}$ 在 $\mathrm{CO}$ 饱和的 $0.5 \mathrm{~mol} / \mathrm{L}$ $\mathrm{H}_{2} \mathrm{SO}_{4}$ 中的循环伏安曲线 ${ }^{[42]}$

Fig. $2 \mathrm{CV}$ curves of $\mathrm{Pt} / \mathrm{C}(\mathrm{Pt} / \mathrm{E}-\mathrm{Tek}), \mathrm{Pt} / \mathrm{HDP}$ and $\mathrm{Pt} / \mathrm{ODP}$ in $\mathrm{CO}_{\text {ads }} 0.5 \mathrm{~mol} / \mathrm{L} \mathrm{H}_{2} \mathrm{SO}_{4}{ }^{[42]}$

剂的 ESA, 同时 Pt-NPs 粒径不能太小, 其粒径范围 为 $50 \sim 350 \mathrm{~nm}$ 。

\section{3 非掺杂的纳米金刚石为载体}

非掺杂的 ND 实际上也具有一定的导电性, 不 过其导电机理与 BDD 不同。Zang 等 ${ }^{[44-45]}$ 很早就开 始研究非掺杂 ND 的电化学性能, 并发现 ND 表面 缺陷和吸附的官能团使其具有一定导电性, 因此高 稳定性的 ND 也可以做铂基催化剂的载体。他们利 用微波乙二醇还原法制备了 $\mathrm{Pt} / \mathrm{ND}$, 并发现其对
MOR 具有较高催化活性, 且稳定性高于 $\mathrm{Pt} / \mathrm{C}$ 。ND 的粒径尺寸影响催化剂的催化活性, $\mathrm{Lu}$ 等 ${ }^{[46]}$ 研究认 为粒径为 $5 \mathrm{~nm}$ 的 $\mathrm{ND}$ 负载的 $\mathrm{PtRu}$ 合金催化剂的活 性最高。同时对 ND 进行表面改性处理, 在保持其 出色稳定性的前提下，提高它的导电性及其与 Pt-NPs 之间的结合力，或者降低阳极反应副产物的 毒化作用，可使 ND 更适合做催化剂的载体材料。 具体的改性方法包括: (1)对 ND 进行真空热处理使其 表面形成几纳米厚的石墨烯层，获得核壳结构的石 墨化金刚石 $(\mathrm{ND} @ \mathrm{G})^{[47]}$; (2)在 ND 表面进行负载或者 镀覆处理，使其表面形成过渡族金属化合物(TMC) 纳米层, $\mathrm{TMC}$ 主要包括 $\mathrm{TiO}_{2} 、 \mathrm{TiN} 、 \mathrm{TiC}$ 等 ${ }^{[48-50]}$ 。

与 $\mathrm{Pt} / \mathrm{ND}$ 相比, $\mathrm{Pt} / \mathrm{ND} @ \mathrm{G}$ 对 MOR 的催化活性 显著提高, 氧化峰电流增大近一倍。通过计时电流 测试, Zang 等 ${ }^{[47]}$ 发现 Pt/ND@G 的催化电流比 Pt/ND 衰减的缓慢, 展现出更好的稳定性。Pt/ND@G 也可 用于催化 ORR, 其稳定性也十分出色。Dong 等 ${ }^{[51]}$ 发 现真空热处理温度在一定程度上影响 $\mathrm{Pt} / \mathrm{ND} @ \mathrm{G}$ 的 ORR 催化活性的稳定性, 通过加速老化试验(ADT), $\mathrm{Pt} / \mathrm{ND} @ \mathrm{G}-1600\left(1600{ }^{\circ} \mathrm{C}\right.$ 热处理)和 Pt/ND@G-1300 $\left(1300^{\circ} \mathrm{C}\right.$ 热处理) 的 $\mathrm{ESA}$ 还剩下初始值的 $67 \%$ 和 $35 \%$, 而 $\mathrm{Pt} / \mathrm{C}$ 仅剩下初始值的 5\%, 如图 3(a)所示。ADT 前后催化 ORR 的半波电位的变化情况如图 3(b)所 示, 其中 Pt/ND@G-1600 的半波电位左移了19 mV, 而 Pt/ND@G-1300 和 Pt/C 则左移了 53 和 $116 \mathrm{mV}$ 。 这充分说明 Pt/ND@G-1600 的 ORR 催化活性下降 最为缓慢, 稳定性最好。与此类似, $\mathrm{Pt} / \mathrm{TiO}_{2} / \mathrm{ND}$ 、 $\mathrm{Pt} / \mathrm{TiN} / \mathrm{ND}$ 和 $\mathrm{Pt} / \mathrm{TiC} / \mathrm{ND}$ 都表现出远优于 $\mathrm{Pt} / \mathrm{C}$ 的稳 定性, 这主要归功于高稳定性的 ND 为内核, 石墨 烯层和表面的 TMC 层也都起到了提高导电性和针 定 Pt-NPs 的作用。
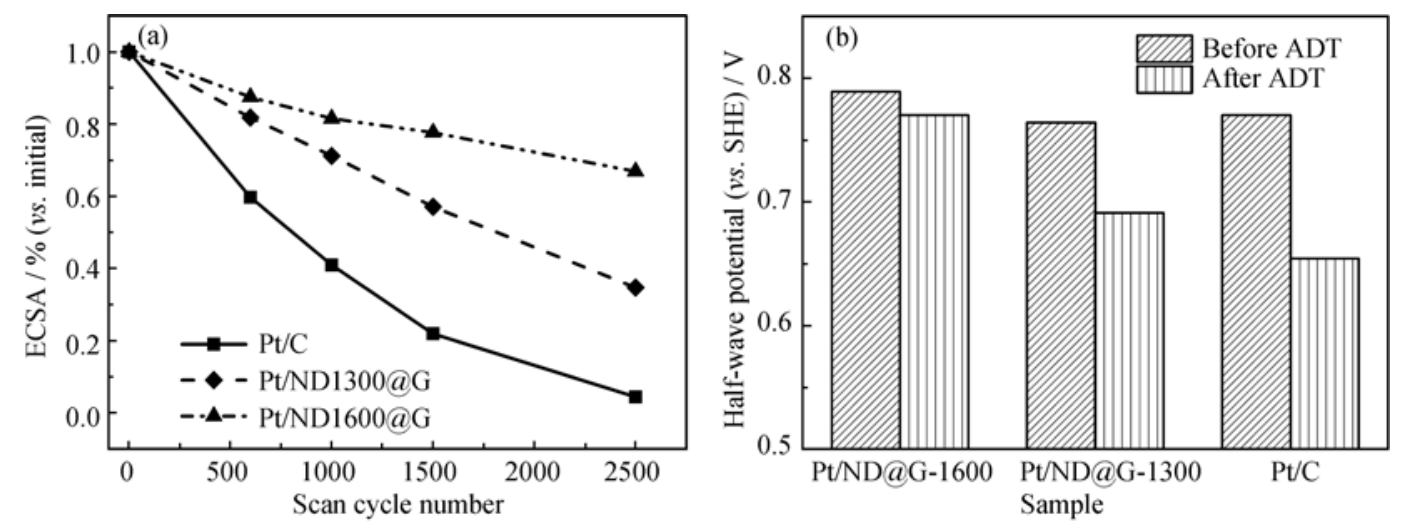

图 3 (a)Pt/C、Pt/ND@G-1300 和 Pt/ND@G-1600 在 ADT 过程中 ESA 变化情况对比图, (b)ADT 完成前后三种催化剂的半波电 位变化情况 ${ }^{[51]}$

Fig. 3 (a) Changes of Pt/C, Pt/ND@G-1300 and Pt/ND@G-1600 ESA related (vs. initial) with cycle number, (b) half-wave potentials of $\mathrm{Pt} / \mathrm{GND} 1300, \mathrm{Pt} / \mathrm{GND} 1600$ and $\mathrm{Pt} / \mathrm{C}$ before and after $\mathrm{ADT}^{[51]}$ 
此外, $\mathrm{Pt} / \mathrm{TiO}_{2} / \mathrm{ND} 、 \mathrm{Pt} / \mathrm{TiN} / \mathrm{ND}$ 和 $\mathrm{Pt} / \mathrm{TiC} / \mathrm{ND}$ 也展 现了比 $\mathrm{Pt} / \mathrm{ND}$, 甚至 $\mathrm{Pt} / \mathrm{C}$ 更高的 MOR 催化活性 ${ }^{[48-50]}$ 。 这主要是因为 $\mathrm{Ti}$ 可以形成 $\mathrm{Ti}-\mathrm{OH}$ 基团, $\mathrm{Ti}-\mathrm{OH}$ 可以 有助于移除甲醇氧化过程中产生的中间产物, 提高 Pt-NPs 的抗 CO 中毒能力。

\section{2 金刚石基非铂催化剂}

Liu 等 ${ }^{[52]}$ 利用光刻及等离子腐蚀技术, 首先制 备得到 $\mathrm{Si}$ 微纳米线, 然后以其为硬模板, 通过 CVD 法制备得到 $\mathrm{B} 、 \mathrm{~N}$ 共掺杂纳米金刚石阵列(VA-BND), 如图 4 所示。VA-BND 能够催化 $0.1 \mathrm{~mol} / \mathrm{L} \mathrm{KOH}$ 溶 液中的 MOR, 其 MOR 氧化峰值电位 $-0.12 \mathrm{~V}$, 低于 $\mathrm{Pt} / \mathrm{C}$ 的-0.02 V, 说明其在碱性环境中的 MOR 催化 活性比 $\mathrm{Pt} / \mathrm{C}$ 更高。同时 VA-BND 对 MOR 的催化活 性也远好于 VA-NDD 和 VA-BDD。B 掺杂引起 VA-BND 表面电荷分布不均, $\mathrm{B}$ 以及 $\mathrm{N}$ 原子附近的 $\mathrm{C}$ 均显正电性, 对 MOR 中的第 1、2 步基元反应有较 强催化活性。 $\mathrm{B} 、 \mathrm{~N}$ 共掺杂提高了掺杂密度, 因此催 化活性明显优于VA-NDD 和 VA-BDD。在 CA 测试 中, VA-BND 也表现出极好的电化学稳定性, MOR 催化电流没有明显衰减。Gan 等 ${ }^{[53]}$ 采取滴落涂布法 将酞菁铜微晶修饰到氢终止和氧终止的 BDD 薄膜 表面, 通过电化学测试分析发现酞菁铜微晶修饰氢 终止 BDD 对 ORR 的催化活性优于氧终止 BDD, 不 过催化的 ORR 主要是以 2 电子形式进行。Koh 等 ${ }^{[54]}$ 以 $5 \mathrm{~nm}$ 的 ND 为基础材料, 利用真空热处理技术使 其表面碳化, 形成石墨烯层, 然后, 使用改进的 Hummer 法腐蚀石墨烯层使其破裂, 并与三聚氰胺 混合热处理, 对破裂的石墨烯层实现掺氮 (N-exhND)。N-exhND 比 $\mathrm{Pt} / \mathrm{C}$ 催化 $\mathrm{ORR}$ 的半坡电 位仅差 $51 \mathrm{mV}$, 同时 N-exhND 具有明显的抗甲醇能 力。通过 5000 圈 ADT 以后, N-exhND 催化 ORR 的 半坡电位只左移 $31 \mathrm{mV}$, 而 $\mathrm{Pt} / \mathrm{C}$ 却左移 $133 \mathrm{mV}$, N-exhND 的稳定性优势明显。
Dong 等 ${ }^{[55]}$ 利用浓 $\mathrm{H}_{2} \mathrm{SO}_{4}$ 和浓 $\mathrm{HNO}_{3}$ 对石墨化 $\mathrm{ND}$ 表面的石墨烯层进行腐蚀处理, 使石墨结构中 出现更多缺陷, 然后引入三聚氧胺进行热处理, 最 终得到氮掺杂的石墨化 ND(N-C/ND)。ADT 结果证 明 N-C/ND 的高稳定性十分优异, ND 为核芯应该是 保证催化剂稳定性的关键因素。在 ND 的石墨化过 程中可以尝试掺杂 $\mathrm{N} 、 \mathrm{~B}$, 只是 ND 的石墨化温度 较高(高于 $1200^{\circ} \mathrm{C}$ ), B-C、C-N 键在高温环境下不稳 定 $\left(1000^{\circ} \mathrm{C}\right)$ 。 $\mathrm{Liu}$ 等 ${ }^{[56]}$ 采用 $\mathrm{FeCl}_{3}$ 为催化剂, 降低了 $\mathrm{ND}$ 的石墨化温度, 并在其石墨化过程引入 $\mathrm{B} 、 \mathrm{~N}$ 源, 实现 $\mathrm{B} 、 \mathrm{~N}$ 共掺杂, 即一步法对石墨化 ND 完成了 掺杂。B、N 双掺杂石墨化 ND 在碱性环境中对 ORR 催化活性较高, 且稳定性出色。 $\mathrm{Wu}$ 等 ${ }^{[57]}$ 则制备了 Co、 $\mathrm{N}$ 共掺的石墨化 ND(Co-N-C/ND), 在碱性环境 下, Co-N-C/ND 催化 ORR 主要以四电子方式进行, 活性接近 $\mathrm{Pt} / \mathrm{C}$, 两者催化 $\mathrm{ORR}$ 的起始电位和半坡电 位基本相同。以计时电流( $(\mathrm{I}-\mathrm{t})$ 方式进行的 $\mathrm{ADT}$ 测试 结果如 5 图所示, 与以 XC-72 为核的 Co-N-C 对比, Co-N-C/ND 的催化活性更加稳定。这充分证明 ND 核芯对催化剂稳定性的重要作用。Zhu 等 ${ }^{[58]}$ 也制备 了 $\mathrm{N}-\mathrm{C} / \mathrm{ND}$, 并注重分析了 ND核芯的重要作用, 他 们通过调整热处理温度, 获得不同比例的 $\mathrm{sp}^{2}$ 和 $\mathrm{sp}^{3}$ 碳, 即石墨化程度不同的 N-C/ND。适当提高 $\mathrm{sp}^{2}$ 碳比例能 够提高催化 ORR 的活性。通过比较稳定性测试前后 $\mathrm{sp}^{2}$ 和 $\mathrm{sp}^{3}$ 碳比例变化, 他们提出在稳定性测试过程 中, $\mathrm{ND}$ 中的 $\mathrm{sp}^{3}$ 碳转化为 $\mathrm{sp}^{2}$ 碳, $\mathrm{sp}^{3} \mathrm{C}-\mathrm{N}$ 键转变为 $\mathrm{sp}^{2} \mathrm{C}-\mathrm{N}$ 键, 这不但增加了导电性, 也增加了催化活 性点, 进而在机理上解释了这一特殊结构的独特优 势。此外, 韩国研究者 Jang 等 ${ }^{[59]}$ 利用激光辐射技术, 将 $5 \mathrm{nmND}$ 石墨化温度降低至 $800^{\circ} \mathrm{C}$, 并在石墨化过 程中引入 $\mathrm{NH}_{3}$ 做氮源实现氮掺杂, 引入 $\mathrm{H}_{2} \mathrm{~S}$ 做硫源 实现硫掺杂。他们还讨论了在辐照过程中使用乙醇和 己醇两种不同分散液对最终石墨化程度的影响。两种 催化剂均展现出较高的 ORR 催化活性, 但氮掺杂催 化活性更高, 催化的 ORR 主要以四电子方式进行。

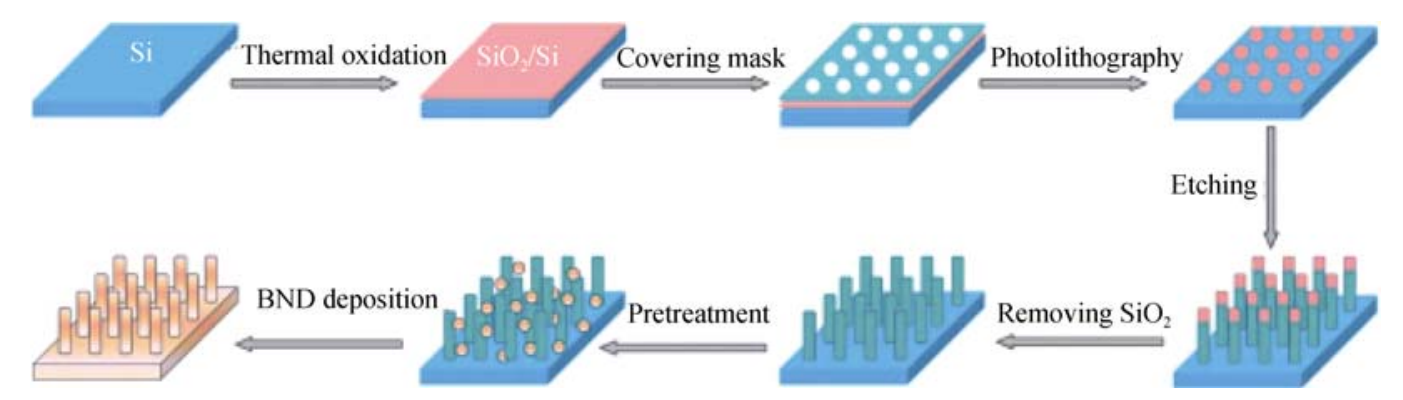

图 4 VA-BND 制备流程示意图 ${ }^{[52]}$

Fig. 4 The procedures for VA-BND preparation ${ }^{[52]}$ 


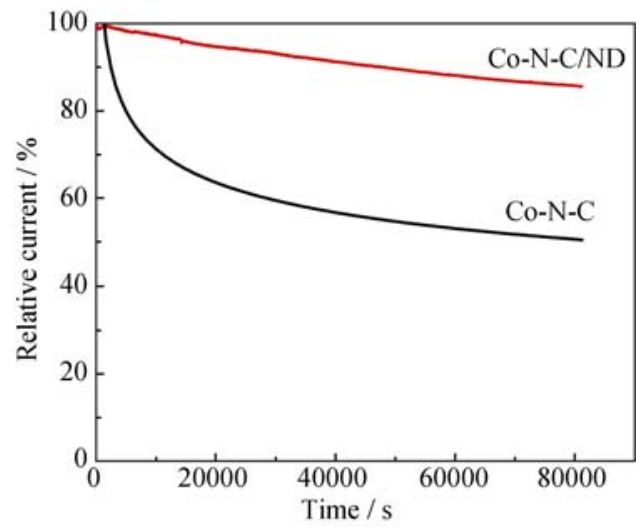

图 $5 \mathrm{Co}-\mathrm{N}-\mathrm{C} / \mathrm{ND}$ 与 $\mathrm{Co}-\mathrm{N}-\mathrm{C}$ 在氧饱和 $0.1 \mathrm{~mol} / \mathrm{L} \mathrm{KOH}$ 溶液中 的 I- $t$ 对比曲线 ${ }^{[57]}$

Fig. 5 I- $t$ curves for $\mathrm{Co}-\mathrm{N}-\mathrm{C} / \mathrm{ND}$ and $\mathrm{Co}-\mathrm{N}-\mathrm{C}$ in $\mathrm{O}_{2}$-saturated $0.1 \mathrm{~mol} / \mathrm{L} \mathrm{KOH}$ solution ${ }^{[57]}$

\section{3 结束语}

以金刚石为载体, 能够从防止结构退化塌陷、 抗 CO 中毒、提高与 Pt-NPs 结合力等方面提高铂基 催化剂的稳定性。特别是通过 TMC 表面修饰、氢 终止或者氧终止处理、表面石墨化等手段对金刚石 进行处理, 可以进一步扩大其做载体的优势。掺杂 $\mathrm{B} 、 \mathrm{~N}$ 等是制备金刚石基非铂催化剂的主要手段, 既 包括向金刚石晶格中掺杂，也包括向金刚石衍生的 石墨结构中进行掺杂。同时掺杂处理并不影响金刚 石的抗氧化能力, 而且 $\mathrm{sp}^{3}$ 碳在电催化过程中有转 化为 $\mathrm{sp}^{2}$ 碳的可能, 因此极强的稳定性也是金刚石 基非铂催化剂的主要优势。

展望未来, 引入新型表面修饰层或者表面官能 团进一步提高金刚石基铂催化剂的催化活性和稳定 性仍将是该方向的重点研究内容, 特别是可控性 强、制备方法绿色简单的改性处理技术将更为受关 注。金刚石基非铂催化剂的研究工作开展时间较短, 整个领域方兴未艾, 无论是从催化剂机理、稳定性 机理以及制备技术方面都具有较大的研究空间，而 且与其它材料进行复合, 研发新型非铂催化剂也可 能成为热门研究方向。总之, 金刚石在然料电池领 域还具有巨大的待发掘潜力。

\section{参考文献:}

[1] 黄镇江, 刘风君. 燃料电池及其应用. 北京: 电子工业出版社, 2005: 5-14

[2] SHARAF O Z, ORHAN M F. An overview of fuel cell technology: fundamentals and applications. Renew. Sust. Energ. Rev., 2014, 32: $810-853$.

[3] O'HAYRE R, CHA S W, PRINZ F B, et al. Fuel Cell
Fundamentals. The second edition. New York: John Wiley \& Sons, 2016: 3-23.

[4] ARICO A, SRINIVASAN S, ANTONUCCI V. DMFCs: From fundamental aspects to technology development. Fuel cells, 2001, 1(2): 133-161.

[5] MCNICOL B, RAND D, WILLIAMS K. Direct methanol-air fuel cells for road transportation. J. Power Sources, 1999, 83(1): 15-31.

[6] YU X, YE S. Recent advances in activity and durability enhancement of $\mathrm{Pt} / \mathrm{C}$ catalytic cathode in PEMFC. J. Power Sources, 2007, 172(1): 133-144.

[7] KANGASNIEMI K H, CONDIT D, JARVI T. Characterization of vulcan electrochemically oxidized under simulated pem fuel cell conditions. J. Electrochem. Soc., 2004, 151(4): E125-E132.

[8] REISER C A, BREGOLI L, PATTERSON T W, et al. A reversecurrent decay mechanism for fuel cells. Electrochem. Solid-State Lett., 2005, 8(6): A273-A276.

[9] LIU M, CHEN W. Green synthesis of silver nanoclusters supported on carbon nanodots: enhanced photoluminescence and high catalytic activity for oxygen reduction reaction. Nanoscale, 2013, 5(24): 12558-12564.

[10] SHAO Y, YIN G, GAO Y, et al. Durability study of PtC and PtCNTs catalysts under simulated pem fuel cell conditions. $J$. Electrochem. Soc., 2006, 153(6): A1093-A1097.

[11] LIU M, LU Y, CHEN W. PdAg nanorings supported on graphene nanosheets: highly methanol - tolerant cathode electrocatalyst for alkaline fuel cells. Adv. Funct. Mater., 2013, 23(10): 1289-1296.

[12] LIU M, ZHANG R, CHEN W. Graphene-supported nanoelectrocatalysts for fuel cells: synthesis, properties, and applications. Chem. Rev., 2014, 114(10): 5117-5160.

[13] LU Y, JIANG Y, WU H, et al. Nano-PtPd cubes on graphene exhibit enhanced activity and durability in methanol electrooxidation after Co stripping-cleaning. J. Phys. Chem. C, 2013, 117(6): 2926-2938.

[14] LU Y, JIANG Y, CHEN W. Graphene nanosheet-tailored ptpd concave nanocubes with enhanced electrocatalytic activity and durability for methanol oxidation. Nanoscale, 2014, 6(6): 3309-3315.

[15] ZHANG R, CHEN W. Non-Precious Ir-V bimetallic nanoclusters assembled on reduced graphene nanosheets as catalysts for the oxygen reduction reaction. J. Mater. Chem. A, 2013, 1(37): 11457-11464.

[16] ZHANG R, HE S, LU Y, et al. Fe, Co, N-functionalized carbon nanotubes in situ grown on $3 \mathrm{~d}$ porous $\mathrm{N}$-doped carbon foams as a noble metal-free catalyst for oxygen reduction. J. Mater. Chem. A, 2015, 3(7): 3559-3567.

[17] LIU M, HE S, CHEN W. Free-standing 3D hierarchical carbon foam-supported PtCo nanowires with "Pt Skin" as advanced 
electrocatalysts. Electrochim. Acta, 2016, 199: 218-226.

[18] 方啸虎. 超硬材料科学与技术. 北京: 中国建材工业出版社, 1998: 17-21.

[19] AVGOUROPOUlos G, IOANNIDES T. CO Tolerance of Pt and Rh catalysts: effect of co in the gas-phase oxidation of $\mathrm{H}_{2}$ over Pt and Rh supported catalysts. Appl. Catal. B- Environ., 2005, 56(1): $77-86$.

[20] GARC A G, SILVA-CHONG J, GUILL N-VILLAFUERTE O, et al. $\mathrm{CO}$ tolerant catalysts for PEM fuel cells: spectroelectrochemical studies. Catal. Today, 2006, 116(3): 415-421.

[21] FERREIRA P, SHAO-HORN Y, MORGAN D, et al. Instability of $\mathrm{PtC}$ electrocatalysts in proton exchange membrane fuel cells a mechanistic investigation. J. Electrochem. Soc., 2005, 152(11): A2256-A2271.

[22] KOH S, YU C, MANI P, et al. Activity of ordered and disordered pt-co alloy phases for the electroreduction of oxygen in catalysts with multiple coexisting phases. J. Power Sources, 2007, 172(1): $50-56$.

[23] BAR-ON I, KIRCHAIN R, ROTH R. Technical cost analysis for pem fuel cells. J. Power Sources, 2002, 109(1): 71-75.

[24] GONG K, DU F, XIA Z, et al. Nitrogen-doped carbon nanotube arrays with high electrocatalytic activity for oxygen reduction. Science, 2009, 323(5915): 760-764.

[25] QU L, LIU Y, BAEK J B, et al. Nitrogen-doped graphene as efficient metal-free electrocatalyst for oxygen reduction in fuel cells. ACS Nano, 2010, 4(3): 1321-1326.

[26] KRAMM U I, LEF VRE M, LAROUCHE N, et al. Correlations between mass activity and physicochemical properties of fe/n/c catalysts for the ORR in PEM fuel cell via ${ }^{57} \mathrm{Fe}$ mößbauer spectroscopy and other techniques. J. Am. Chem. Soc., 2013, 136(3): 978-985.

[27] SWAIN G M. The Susceptibility to surface corrosion in acidic fluoride media: a comparison of diamond, hopg, and glassy carbon electrodes. J. Electrochem. Soc., 1994, 141(12): 3382-3393.

[28] GAO F, YANG N, SMIRNOV W, et al. Size-controllable and homogeneous platinum nanoparticles on diamond using wet chemically assisted electrodeposition. Electrochim. Acta, 2013, 90: 445-451.

[29] LU X, HU J, FOORD J S, et al. Electrochemical deposition of $\mathrm{Pt}-\mathrm{Ru}$ on diamond electrodes for the electrooxidation of methanol. J. Electroanal. Chem., 2011, 654(1): 38-43.

[30] MAVROKEFAlOS C K, Nelson G W, POLL C G, et al. Electrochemical aspects of $\mathrm{Pt}-\mathrm{Cu}$ and $\mathrm{Cu}$ modified boron-doped diamond. Phys. Status Solidi A, 2015, 212(11): 2559-2567.

[31] SIN G, FOTI G, COMNINELLIS C. Boron-doped diamond (bdd)-supported $\mathrm{pt} / \mathrm{sn}$ nanoparticles synthesized in microemulsion systems as electrocatalysts of ethanol oxidation. J. Electroanal.
Chem., 2006, 595(2): 115-124.

[32] SALAZAR-BANDA G R, SUFFREDINI H B, AVACA L A, et al. methanol and ethanol electro-oxidation on $\mathrm{Pt}-\mathrm{SnO}_{2}$ and $\mathrm{Pt}_{-}-\mathrm{Ta}_{2} \mathrm{O}_{5}$ Sol-Gel-modified boron-doped diamond surfaces. Mater. Chem. Phys., 2009, 117(2): 434-442.

[33] WANG J, SWAIN G, TACHIBANA T, et al. The incorporation of Pt nanoparticles into boron-doped diamond thin-films: dimensionally stable catalytic electrodes. J. New Mat. Electr. Sys, 2000, 3(1): $75-82$.

[34] LYU X, HU J, FOORD J S, et al. A novel electroless method to prepare a platinum electrocatalyst on diamond for fuel cell applications. J. Power Sources, 2013, 242: 631-637.

[35] GONZALEZ-GONZALEZ I, TRYK D, CABRERA C R. Polycrystalline boron-doped diamond films as supports for methanol oxidation electrocatalysts. Diam. Relat. Mater., 2006, 15(2): $275-278$.

[36] EL ROUSTOM B, SINE G, FOTI G, et al. A novel method for the preparation of bi-metallic (Pt-Au) nanoparticles on boron doped diamond (BDD) substrate: application to the oxygen reduction reaction. J. Appl. Electrochem., 2007, 37(11): 1227-1236.

[37] SPĂTARU T, PREDA L, OSICEANU P, et al. Electrochemical deposition of $\mathrm{Pt}-\mathrm{RuO}_{x} \mathrm{Nh}_{2} \mathrm{O}$ composites on conductive diamond and its application to methanol oxidation in acidic media. Electrocatalysis, 2016, 7(2): 140-148.

[38] SALAZAR-BANDA G R, EGUILUZ K I, AVACA L A. Boron-doped diamond powder as catalyst support for fuel cell applications. Electrochem. Commun., 2007, 9(1): 59-64.

[39] SPĂTARU N, ZHANG X, SPĂTARU $T$, et al. Platinum electrodeposition on conductive diamond powder and its application to methanol oxidation in acidic media. J. Electrochem. Soc., 2008, 155(3): B264-B269.

[40] LA-TORRE-RIVEROS L, ABEL-TATIS E, M NDEZ-TORRES A E, et al. Synthesis of platinum and platinum-ruthenium-modified diamond nanoparticles. J. Nanopart. Res., 2011, 13(7): 2997-3009.

[41] KIM J, CHUN Y S, LEE S K, et al. Improved electrode durability using a boron-doped diamond catalyst support for proton exchange membrane fuel cells. RSC Advances, 2015, 5(2): 1103-1108.

[42] CELORRIO V, PLANA D, FL REZ-MONTA O J, et al. Methanol oxidation at diamond-supported Pt nanoparticles: effect of the diamond surface termination. J. Phys. Chem. C, 2013, 117(42): 21735-21742.

[43] WANG J, SWAIN G M. Fabrication and evaluation of platinum/diamond composite electrodes for electrocatalysis preliminary studies of the oxygen-reduction reaction. $J$. Electrochem. Soc., 2003, 150(1): E24-E32.

[44] ZANG J, WANG Y, ZHAO S, et al. Electrochemical properties of nanodiamond powder electrodes. Diam. Relat. Mater., 2007, 16(1): 
16-20.

[45] BIAN L, WANG Y, ZANG J, et al. Microwave synthesis and characterization of pt nanoparticles supported on undoped nanodiamond for methanol electrooxidation. Int. J. Hydrogen. Energ, 2012, 37(2): 1220-1225.

[46] LU R, ZANG J, WANG Y, et al. Microwave synthesis and properties of nanodiamond supported ptru electrocatalyst for methanol oxidation. Electrochim. Acta, 2012, 60: 329-333.

[47] ZANG J, WANG Y, BIAN L, et al. Graphene growth on nanodiamond as a support for a Pt electrocatalyst in methanol electro-oxidation. Carbon, 2012, 50(8): 3032-3038.

[48] ZHAO Y, WANG Y, CHENG X, et al. Platinum nanoparticles supported on epitaxial $\mathrm{TiC} /$ nanodiamond as an electrocatalyst with enhanced durability for fuel cells. Carbon, 2014, 67: 409-416.

[49] ZHAO Y, WANG Y, DONG L, et al. Core-shell structural nanodiamond@tin supported pt nanoparticles as a highly efficient and stable electrocatalyst for direct methanol fuel cells. Electrochim. Acta, 2014, 148: 8-14.

[50] ZHAO Y, WANG Y, ZANG J, et al. A novel support of nano titania modified graphitized nanodiamond for Pt electrocatalyst in direct methanol fuel cell. Int. J. Hydrogen. Energy, 2015, 40(13): $4540-4547$.

[51] DONG L, ZANG J, WANG Y, et al. Graphitized nanodiamond as highly efficient support of electrocatalysts for oxygen reduction reaction. J. Electrochem. Soc., 2014, 161(3): F185-F191.

[52] LIU Y, CHEN S, QUAN X, et al. Tuning the electrochemical properties of a boron and nitrogen codoped nanodiamond rod array to achieve high performance for both electro-oxidation and electro-reduction. J. Mater. Chem. A, 2013, 1(46): 14706-14712.

[53] GAN P, FOORD J S, COMPTON R G. Surface modification of boron-doped diamond with microcrystalline copper phthalocyanine: oxygen reduction catalysis. Chemistry Open, 2015, 4(5): 606-612.

[54] KOH J, PARK S H, CHUNG M W, et al. Diamond@ carbon-onion hybrid nanostructure as a highly promising electrocatalyst for the oxygen reduction reaction. RSC Advances, 2016, 6(33): $27528-27534$.

[55] DONG L, ZANG J, SU J, et al. Nanodiamond/ nitrogen-doped graphene (core/shell) as an effective and stable metal-free electrocatalyst for oxygen reduction reaction. Electrochim. Acta, 2015, 174: 1017-1022.

[56] LIU X, WANG Y, DONG L, et al. One-step synthesis of shell/core structural boron and nitrogen co-doped graphitic carbon/ nanodiamond as efficient electrocatalyst for the oxygen reduction reaction in alkaline media. Electrochim. Acta, 2016, 194: 161-167.

[57] WU Y, ZANG J, DONG L, et al. High performance and bifunctional cobalt-embedded nitrogen doped carbon/nanodiamond electrocatalysts for oxygen reduction and oxygen evolution reactions in alkaline media. J. Power Sources, 2016, 305: 64-71.

[58] ZHU Y, LIN Y, ZHANG B, et al. Nitrogen-doped annealed nanodiamonds with varied $\mathrm{Sp} 2 / \mathrm{Sp} 3$ ratio as metal - free electrocatalyst for the oxygen reduction reaction. ChemCatChem, 2015, 7(18): 2840-2845.

[59] JANG D M, IM H S, BACK S H, et al. Laser-induced graphitization of colloidal nanodiamonds for excellent oxygen reduction reaction. Phys. Chem. Chem. Phys., 2014, 16(6): 2411-2416. 